\title{
Transition from static to kinetic friction in a model lubricated system
}
A. Levent DemirelSteve Granick

Citation: The Journal of Chemical Physics 109, 6889 (1998); doi: 10.1063/1.477256

View online: http://dx.doi.org/10.1063/1.477256

View Table of Contents: http://aip.scitation.org/toc/jcp/109/16

Published by the American Institute of Physics

\section{Articles you may be interested in}

The Nature of the Static and Kinetic Coefficients of Friction Journal of Applied Physics 22, 1373 (2004); 10.1063/1.1699869

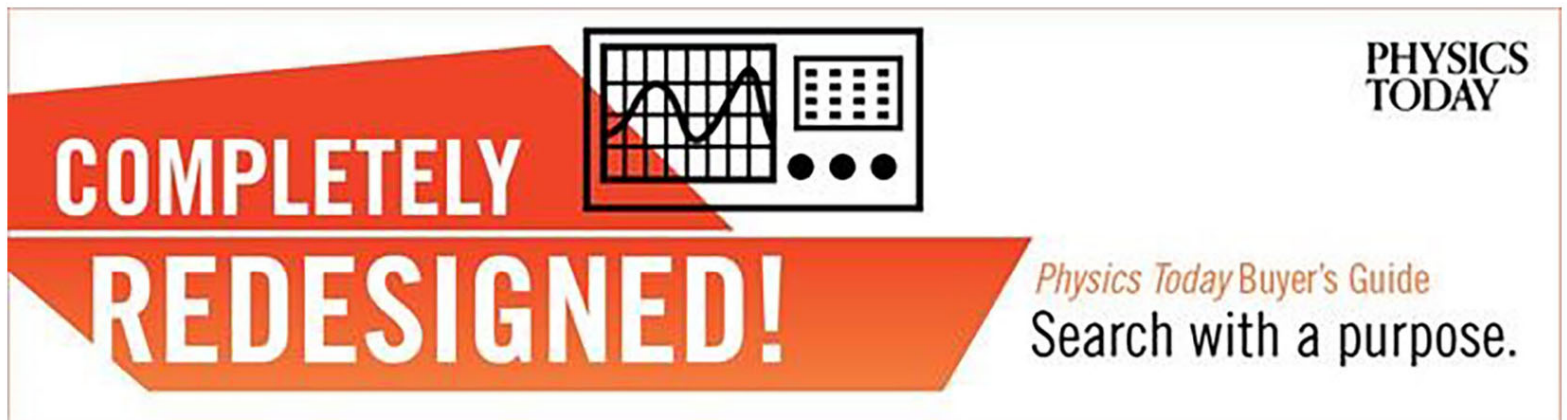




\title{
Transition from static to kinetic friction in a model lubricated system
}

\author{
A. Levent Demirel \\ Chemistry Department, Koç University, Çayir Cad. No: 5 Istinye 80860 Istanbul, Turkey \\ Steve Granick ${ }^{\mathrm{a})}$ \\ Department of Materials Science and Engineering, University of Illinois, Urbana, Illinois 61801
}

(Received 18 May 1998; accepted 24 July 1998)

\begin{abstract}
Molecularly thin confined fluids were deformed in shear faster than structural relaxations in response to shear could be accomplished, such that with increasing deformation the systems passed from the rest state to sliding. The response of these systems - two atomically smooth mica sheets separated by a fluid comprised of globularly shaped molecules [octamethylcyclotetrasiloxane] —was studied as a function of film thickness of the fluid (from 80 to $10 \AA$, i.e, from $\sim 8$ to $\sim 1$ molecular dimensions), as a function of normal pressure, and as a function of deformation rate, using a modified surface forces apparatus. Whereas the linear response was always liquid-like provided that the deformation rate was sufficiently slow, a "stick-slip" transition from the rest state to sliding was observed when the deformation rate was large, provided that the oscillatory frequency sufficiently exceeded the inverse intrinsic relaxation time of the confined fluid. This transition was monotonic and reversible without hysteresis for relatively thick films but for thinner films was discontinuous with hysteresis. For films thicker than 3 molecular layers (ML), two length scales in deformation were observed; the films showed nonlinear force-deformation response beginning at a deformation amplitude of $3 \AA$ but in general showed stick slip only when the deformation was larger than this. The critical deformation at the point of stick slip decreased from 9 to $3 \AA$ with increasing normal pressure, indicating diminished plasticity of the confined structures with increasing normal pressure. The critical film thickness of 3 ML correlates with the possibility of one rather than more slip planes. The thinnest films under the highest compressive pressures showed moderate increase of the viscous shear force with increasing effective sliding velocity, but in general the viscous force reached a plateau in which force showed almost no dependence on sliding rate. In interpreting the results in the context of friction, static friction was identified with the elastic stress at rupture and kinetic friction was identified with the limiting maximum observed level of viscous force. After normalizing friction and normal forces by the contact area, the static friction coefficient was found to be 0.44 and the kinetic friction coefficient to be 0.14 . In other words, as the normal pressure increased, the elastic force needed to rupture the system increased more rapidly than the limiting shear stress. The magnitude of the limiting shear stress increased exponentially with decreasing film thickness with a decay length of 1 molecular dimension. This decay length correlates well with the known exponential decay of oscillations in the static force-distance profile. The critical shear amplitude of $3 \AA$, relative to the molecular dimension of $\approx 9 \AA$, is reminiscent of early estimates by Frenkel of the point of instability when planes of atoms slide over one another. (C) 1998 American Institute of Physics. [S0021-9606(98)52540-8]
\end{abstract}

\section{INTRODUCTION}

The mechanical and rheological properties of thin lubricating films are important not only for technological but also for fundamental reasons. ${ }^{1-3}$ For model lubricated systems in which simple liquids are confined between two atomically smooth solid surfaces, the fundamental questions of relaxation mechanisms in the linear-response regime, of dissipation mechanisms in the nonlinear-response regime, and of the transition from linear to nonlinear responses with increasing deformation amplitude, leave their place to technological interest when one recalls that the peak stress in the

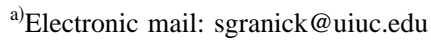

linear-response regime can be identified with the static friction and the nonlinear-response regime can be identified with the sliding (kinetic) friction.

In 1686, Newton introduced a linear relationship ${ }^{4}$ to describe the resistance of fluid flow to the rate of deformation, $\sigma=\eta \dot{\gamma}$, where $\sigma$ is the viscous shear stress, $\dot{\gamma}$ is the shear rate, and the proportionality constant $\eta$ is the viscosity of the fluid, a number that is constant at constant temperature and pressure. This linear relationship holds at low deformation amplitudes, but is not expected to hold for all shear rates; otherwise, the viscous shear stress could increase without limit, an implication that lacks physical sense. At sufficiently large shear rates, nonlinear responses (so called nonNewtonian flows) set in and the viscosity becomes a function of the shear rate. The viscosity decreases with increasing 
shear rate following an apparent power law behavior, $\eta$ $\sim \dot{\gamma}^{-a}(0<a<1)$. Otherwise stated, the shear stress $(\sigma)$ levels off as $\sigma \sim \dot{\gamma}^{1-a}$ (Refs. 5-7). It was initially observed that $a \approx 2 / 3$ for molecularly thin films ${ }^{5}$ but later experiments soon found that $a \rightarrow 1$ with further increases of the sliding velocity. ${ }^{8,9}$ When $a=1$, the viscous stress ceases to show dependence on the shear rate. The exponent itself thus depends on shear rate, and it is now apparent that power-law behavior holds over only a limited range of shear rate.

Nonlinear responses occur when the shear rate $\dot{\gamma}$ exceeds the inverse of the longest structural relaxation time $\tau$ of the fluid. In such a case, the fluid is deformed more rapidly than it can relax and the observed response arises from driven nonequilibrium structures in the fluid. The origin of these structures differs significantly from one system to the other. The distortion might be intramolecular as in the case of long flexible chains: Equilibrium Gaussian chains can become highly stretched under large amplitude shear. ${ }^{5}$ Orientational effects are observed for rigid rod-like molecules and microphase separated block copolymers. ${ }^{10}$ In case of simple fluids, it is the distortion of the fluid structure that gives rise to nonlinear behavior. ${ }^{11}$ The importance of slip for flow in the bulk has been emphasized in polymer rheology ${ }^{12,13}$ and for confined films based on molecular dynamics simulations. $^{14}$

The nonlinear response is easiest to observe experimentally in systems having sluggish relaxation times. Such systems show deviations from linearity at experimentally accessible shear rates, such as polymer melts, ${ }^{5,12,13}$ microphaseseparated block copolymers, ${ }^{15-18}$ worm-like micelles, ${ }^{18,19}$ supercooled liquids, ${ }^{20}$ fluids under high pressure, ${ }^{7}$ and various other concentrated systems including polymer solutions, ${ }^{5}$ colloidal suspensions, ${ }^{21}$ and foams. ${ }^{22}$ The relaxation times of simple liquids in the bulk are of the order of picoseconds and the observation of the onset of nonlinear response is difficult because high shear rates, of the order of $10^{12} \mathrm{~s}^{-1}$, are required. When simple liquids are confined down to molecular dimensions between two solid surfaces, the relaxation time of molecules increase by orders of magnitude $e^{8,23,24}$ and the nonlinear response sets in at significantly lower shear rates. Large strains and shear rates are also easily reached because of very small film thickness (less than $100 \AA$ ). ${ }^{25}$

Friction and boundary lubrication depend significantly on the film thickness of the lubricant (surface separation) and the normal force. Various aspects of friction in molecularly thin liquid films, such as the peak stress in the linearresponse regime (the static friction), the constant value of the stress in the nonlinear-response regime (sliding or kinetic friction) and the transition from static to kinetic friction, have previously been discussed in studies of different liquids. ${ }^{2,3,6,8,9,23-30}$ These works often investigated friction force as a function of the load on the system. It has been well established that short linear chains, including those with one $\mathrm{CH}_{3}$ side group, and globular molecules, form layers parallel to the confining surfaces and that the two surfaces continue to remain separated by an integral number of molecular layers during sliding. However, in most of the previous friction studies, a systematic study of the effect of quantized film thickness has not been discussed.
In this study, we present a systematic investigation of the effect of film thickness on the transition from static to kinetic friction together with the effect of normal pressure. For this purpose, we used globularly shaped molecules [octamethylcyclotetrasiloxane, (OMCTS)] which form layers parallel to the confining surfaces. It was thus possible, below a surface separation of 10 molecular layers (ML), to stabilize the two surfaces separated by integral number of molecular layers.

In an earlier report, we studied the frequency spectrum of OMCTS films in the linear-response regime at different film thickness and showed that these layers presented frequency-dependent viscosity whenever the frequency exceeded a critical frequency whose value diminished with decreasing film thickness, yet Newtonian responses provided that the frequency was sufficiently low. ${ }^{23}$ The point of these experiments was that in a linear-response regime, the equilibrium structure of the film was weakly perturbed by the applied shear, whereas significant structural changes occurred at large oscillation amplitudes. Thus, it was essential to keep the oscillation amplitude small when probing by oscillatory shear the sole effects of confinement on the viscoelastic response. The linear response of molecularly thin OMCTS films was studied systematically as a function of film thickness and oscillation frequency and the existence of a glass-like transition with increasing confinement was shown. $^{23}$

Here, we concentrate on oscillatory shear experiments where the shear rate was changed by changing the oscillation amplitude at constant oscillation frequency.

\section{EXPERIMENT}

The experiments employed a surface forces apparatus modified for oscillatory shear. ${ }^{31}$ A droplet of OMCTS was confined between two atomically smooth curved surfaces of muscovite mica that had been glued onto silica cylinders whose axes were oriented perpendicular to each other. The radius of curvature of the silica lenses was $\approx 2 \mathrm{~cm}$. Below a surface separation of $10 \mathrm{ML}$ (effective monolayers), the glue beneath each mica sheet flattened due to structural forces acting normal to the surfaces, creating a parallel plate geometry. Surface separation was measured using multiple beam interference of white light reflecting between silver coated back sides of mica surfaces. The sensitivity in the measurement of surface separation was $\approx 2-3 \AA$. For normal force measurements, the bottom surface sat on a cantilever leaf spring having a spring constant of $923 \mathrm{~N} / \mathrm{m}$. Oscillatory shear was applied to the top surface using two piezoelectric bimorphs.

A sinusoidal voltage was applied to one of the bimorphs and the response was measured from the other. The resulting sinusoidal applied force, $f(t)=f_{0}$ sin $\omega t$, divided by the total spring constant of the system $k_{T}$, gave the displacement of the top surface with respect to the bottom surface, $x(t)$ $=x_{0} \sin \omega t$, where $x_{0}=f_{0} / k_{T}$. The parameter $k_{T}$ was calculated by modeling the system as having the device stiffness in parallel with the series combination of glue and liquid, as described before. ${ }^{29}$ The velocity of top surface with respect to the bottom one was then $v(t)=v_{0} \cos \omega t$ where velocity amplitude $v_{0}=x_{0} \omega$. The effective strain amplitude was de- 
fined as the ratio of deformation amplitude to the film thickness $h, \gamma=x_{0} / h$, and the effective shear rate as the product of strain and the oscillation frequency, $\dot{\gamma}=\gamma \omega=v_{0} / h$. (We distinguish between actual shear rate and effective shear rate because the latter will exceed the former if the deformation mechanism involves slip, i.e., if strain is not distributed homogeneously through the film.)

The resulting response at the second bimorph was measured by a lock-in amplifier and decomposed into in-phase and out-of-phase components with respect to that when the surfaces were apart. The in-phase component of the resulting stress, normalized by strain, gave the storage modulus, $G^{\prime}$. Similarly the out-of-phase component gave the loss modulus, $G^{\prime \prime}$.

OMCTS (Fluka, purim grade) was used as received after control experiments showed similar behavior following further purification. Experiments were performed at $27^{\circ} \mathrm{C}$. A highly hygroscopic chemical, $\mathrm{P}_{2} \mathrm{O}_{5}$, was kept in the sealed sample chamber throughout the experiment. The sample was left in the dry chamber atmosphere for a few hours before any measurements were done. Surface separation was then decreased slowly. All the data were taken in the direction of decreasing surface separation, so that the system was always in the repulsive force regime, close to the peak of the oscillatory structural forces. Because oscillatory force amplitude increases with decreasing surface separation, below a surface separation of $4 \mathrm{ML}$ it was possible to perform measurements at different increasing values of normal forces while having the same number of molecular layers between the surfaces. At larger separations the repulsive forces were weak, but the systems were transiently stable, long enough to make measurements.

The contact diameter for the cases of smaller film thickness was calculated from the flattened tip of the interference fringes taking into account the lateral magnification of the focusing mechanism. The accuracy in the measurement of contact diameter was $\pm 5 \mu \mathrm{m}$, resulting in considerable error bars in calculating normal pressure and shear stress at surface separations where the contact diameter was approximately equal to or less than $5 \mu \mathrm{m}$. In these instances we calculated the contact diameter with respect to the measured contact diameter of $40 \mu \mathrm{m}$ at the maximum applied normal force of $3.81 \mathrm{mN}$, assuming a Hertzian contact. This is a reasonable assumption when one recalls that it is the glue under the mica surfaces that deforms under the action of normal pressure. The assumption was also justified empirically by the observation that these calculated contact diameters were within $\pm 5 \mu \mathrm{m}$ of the measured values.

\section{RESULTS}

\section{A. Viscoelasticity as a function of film thickness}

In a linear-response regime, the equilibrium structure of the sample is weakly perturbed by the applied deformationbut significant structural changes occur if the deformations are large. Thus, it is essential to keep the deformation amplitude small when studying the sole effects of confinement on the viscoelastic response. ${ }^{5,23}$ Here we study the relation between these linear-response effects and the accompanying

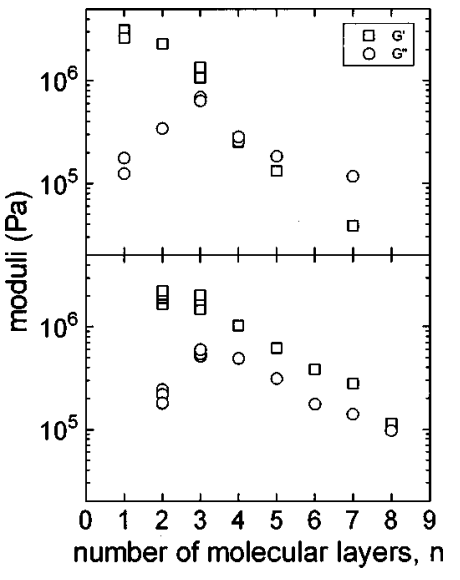

FIG. 1. Linear response of OMCTS films: The effective storage modulus, $G^{\prime}$ (squares) and loss modulus, $G^{\prime \prime}$ (circles) are plotted as a function of film thickness expressed in number of molecular layers between the surfaces. 10 $\mathrm{Hz}$ (top panel); $250 \mathrm{~Hz}$ (bottom panel).

responses of these same systems under large deformations. We show in Fig. 1 the linear viscoelastic response as a function of film thickness at two different oscillation frequencies.

In Fig. 1, the storage modulus $G^{\prime}$ and the loss modulus $G^{\prime \prime}$ are plotted against the number of molecular layers between the confining surfaces at $10 \mathrm{~Hz}$ (top panel) and $250 \mathrm{~Hz}$ (bottom panel) oscillation frequencies. The oscillation amplitude was $4 \AA$ for the thickest films and decreased to $2 \AA$ for the thinnest. The linearity of response was confirmed by Fourier transforming the resulting stress wave forms and by checking the proportionality of the resulting stress amplitude to the amplitude of the applied strain for even larger values of oscillation amplitude. The Fourier transforms showed no significant harmonic components. With increasing confinement, the linear response of OMCTS films went from predominantly viscous $\left(G^{\prime \prime}>G^{\prime}\right)$ to predominantly elastic $\left(G^{\prime}\right.$ $\left.>G^{\prime \prime}\right)$. The crossover film thickness for this transition depended on the oscillation frequency and increased with increasing oscillation frequency. This indicated an increase of the largest relaxation time of the system with confinement as discussed previously in detail. ${ }^{23}$ The crossover film thickness was $4 \mathrm{ML}$ at $10 \mathrm{~Hz}$, as shown in Fig. 1 (top panel). The bottom panel indicates a crossover film thickness of approximately $9 \mathrm{ML}$ at $250 \mathrm{~Hz}$. The monotonic increase of $G^{\prime}$ and $G^{\prime \prime}$ with decreasing film thickness started changing at $3 \mathrm{ML}$. $G^{\prime}$ leveled off and $G^{\prime \prime}$ decreased, resulting in significant elasticity for 1 and 2 ML thick films for which $G^{\prime}$ was approximately an order of magnitude larger than $G^{\prime \prime}$.

\section{B. Transition from linear response (static friction) to nonlinear response (kinetic friction)}

The transition to nonlinear response with increasing oscillation amplitude also showed significant change at a film thickness of $3 \mathrm{ML}$ as shown in Figs. 2-4. For the discussion of nonlinear response, we will only present the data taken at $250 \mathrm{~Hz}$ oscillation frequency. The observations at other oscillation frequencies were similar in the film thickness range where the linear response showed significant elasticity. A sign of nonlinearity is that the resulting stress wave form 


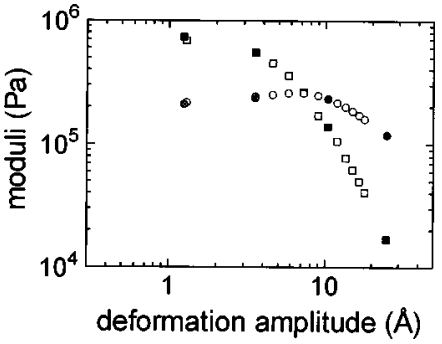

FIG. 2. Gradual transition from linear to nonlinear response, typical of films of thickness $3 \mathrm{ML}$ or more. The effective storage modulus, $G^{\prime}$ (squares) and loss modulus, $G^{\prime \prime}$ (circles) are plotted as a function of the deformation amplitude for a film of thickness $3 \mathrm{ML}$. The solid symbols were taken in the direction of decreasing oscillation amplitude, demonstrating reversibility without hysteresis.

deviates from a pure sine wave and can be expressed as a sum of odd-order harmonics. ${ }^{32}$ For the analysis of the nonlinear response data and the calculation of in-phase and outof-phase components of the resulting stress, we concentrated for reasons of ease of analysis on the response at the fundamental frequency. This is partially justified by the fact that the dissipated energy is completely determined by the fundamental frequency component. ${ }^{32,33}$

Figures 2-4 show forces and normalized forces (the moduli, $G^{\prime}$ and $G^{\prime \prime}$ ) as a function of oscillation amplitude. The data in Fig. 2 correspond to a 3 ML thick film and are typical of even thicker films. In Figs. 3 and 4 (Fig. 3 shows the raw forces, Fig. 4 the normalized forces), the data also correspond to a $3 \mathrm{ML}$ thick film, but under slightly larger normal force. The panels of Fig. 3 and 4 represent 2 ML thick films at varying normal forces indicated in the figure caption and Figs. 3(e) and 4(e), the bottom panels, represents

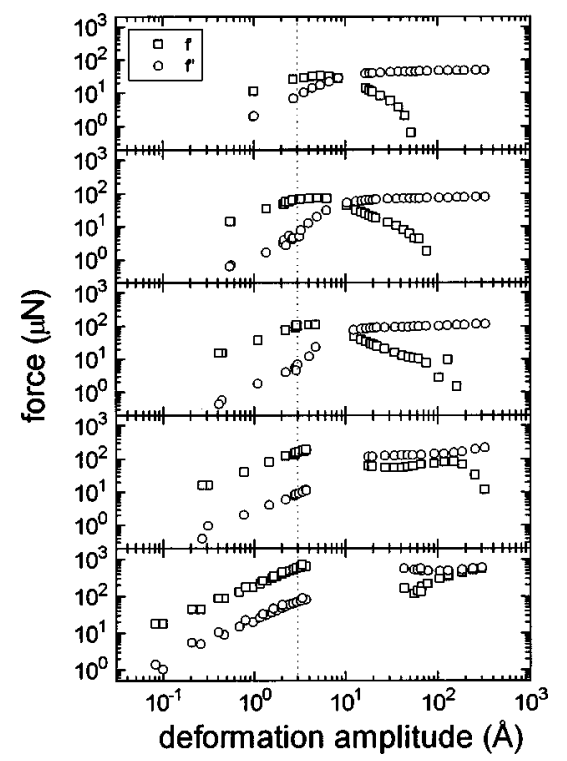

FIG. 3. Discontinuous transition from linear to nonlinear response, typical of films $3 \mathrm{ML}$ or thinner. The elastic force, $f^{\prime}$ (squares) and the dissipative force, $f^{\prime \prime}$ (circles), are plotted against deformation amplitude. The data refer, in sequence from top to bottom, to (a) $3 \mathrm{ML}$ thick film $(n=3)$ at a normal force $\left(F_{n}\right)$ of $0.40 \mathrm{mN}$, (b) $n=2, F_{n}=0.58 \mathrm{mN}$, (c) $n=2, F_{n}=0.75 \mathrm{mN}$, (d) $n=2, F_{n}=1.11 \mathrm{mN}$, (e) $n=1, F_{n}=3.81 \mathrm{mN}$. The dotted vertical line at a deformation amplitude of $3 \AA$ indicates the onset of deviations from constant values of $G^{\prime}$ and $G^{\prime \prime}$ in the linear response regime.

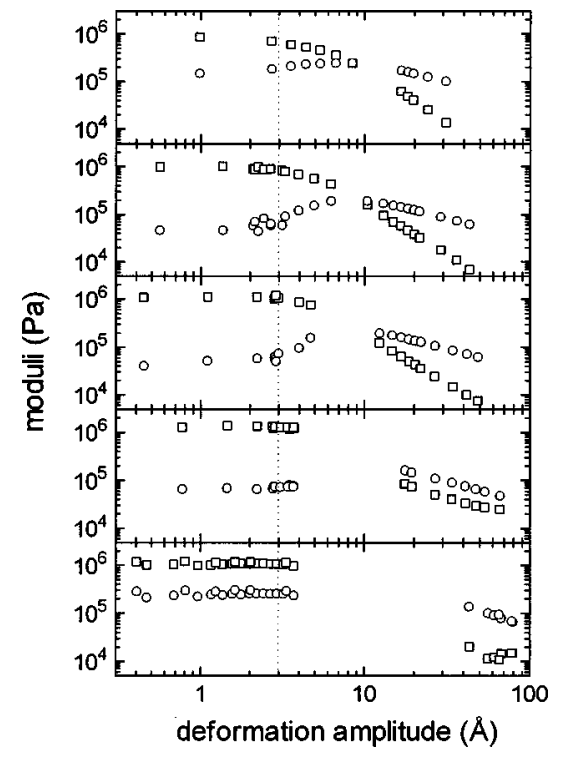

FIG. 4. The data from Fig. 3 are normalized to give the effective storage modulus, $G^{\prime}$ (squares) and loss modulus, $G^{\prime \prime}$ (circles), plotted against the deformation amplitude. In both Figs. 3 and 4.

a single molecular layer of OMCTS between the confining surfaces. Both in Fig. 2 and Figs. 3 and 4, the predominantly elastic linear response at small oscillation amplitudes switched to a predominantly viscous nonlinear response at large oscillation amplitudes. However, this transition for films thicker than 3 ML was significantly different from that for thinner films. The thicker films showed a gradual transition from linear to nonlinear response with increasing oscillation amplitude, as in Fig. 2. The solid symbols were taken in the direction of decreasing oscillation amplitude and both $G^{\prime}$ and $G^{\prime \prime}$ values followed the data points taken in the direction of increasing oscillation amplitude.

The data in Figs. 3 and 4, corresponding to films having significant elasticity with thickness at or below $3 \mathrm{ML}$, show discontinuous transitions from linear to nonlinear response. The discontinuity in the transition started at larger normal forces at a film thickness of $3 \mathrm{ML}$. For these films, the response was also reversible when the oscillation amplitude was decreased from high to low values, but showed hysteresis in the transition region under the largest applied normal forces. It has to be noted that the magnitude of the jump in oscillation amplitude was a function of the spring constant of bimorphs used to shear the upper surface. A larger spring constant would result in a smaller jump in oscillation amplitude at the transition, for obvious reasons. But the discontinuity in $G^{\prime}$ and $G^{\prime \prime}$ was independent of the spring constant. A discontinuous transition and reversibility with significant hysteresis were previously observed for confined branched molecules and for self-assembled monolayers on mica, ${ }^{9,29}$ which led to the conclusion that sliding in the confined liquids is the result of slippage along an interface. The use of OMCTS molecules, in the present study, allowed us to change the film thickness and the normal pressure controllably and to observe the onset of this discontinuous transition with decreasing film thickness (increasing normal pressure). 


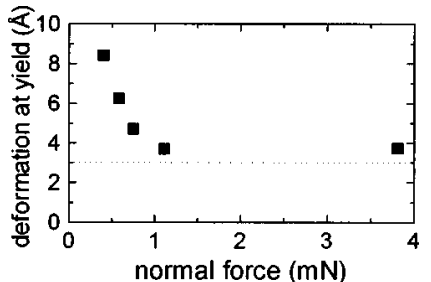

FIG. 5. The deformation amplitude at rupture for the data in Figs. 3 and 4. The dotted line corresponds to the one drawn in Figs. 3 and 4.

\section{Characteristic length scales in discontinuous transitions}

For all elastic structures showing discontinuous transition in Figs. 3 and 4, shear forces increased in strictly linear proportion to the deformation amplitude, provided that the deformations were sufficiently small. Otherwise stated, the magnitudes of the storage and loss moduli were constant. This linear-response region was observed below a deformation amplitude of $3 \AA$, as indicated by the dashed line in Figs. 3 and 4. This characteristic length in deformation amplitude was associated with the onset of yield in the elastic structure and was independent of the film thickness and the normal pressure on the system. Beyond a deformation amplitude of $3 \AA$, the storage modulus decreased and the loss modulus increased until the structure ruptured. The deformation amplitude at rupture is the second characteristic length scale; this decreased with increasing normal pressure as shown in Fig. 5, and approached the deformation amplitude at the onset of yield.

\section{Elastic stress at rupture (static friction)}

The elastic and viscous forces are plotted in Fig. 6 as a function of deformation amplitude for the data in Figs. 3(d) and $4(\mathrm{~d})$. In the linear-response region, force was proportional to the deformation amplitude giving a slope of 1 in the $\log -\log$ plot of Fig. 6. In the region of deformation amplitudes between $3 \AA$ (onset of yield) and rupture, the elastic forces leveled off slightly due to the decreases in $G^{\prime}$. Because stress is the product of $G^{\prime}$ and the deformation amplitude, the decrease in $G^{\prime}$ was partly compensated by increase in deformation amplitude. Therefore, the change in elastic force was not as clear as for $G^{\prime}$ of Fig. 4. The peak elastic force in the linear region (elastic force at rupture) was associated with the static friction. This value increased linearly

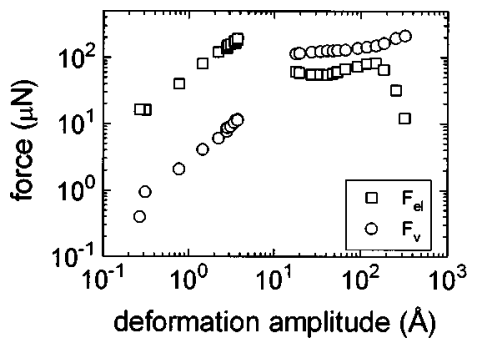

FIG. 6. The elastic and viscous forces for the data in Fig. 4(d), plotted as a function of deformation amplitude. We identify the peak elastic force in the linear response regime with the static friction.
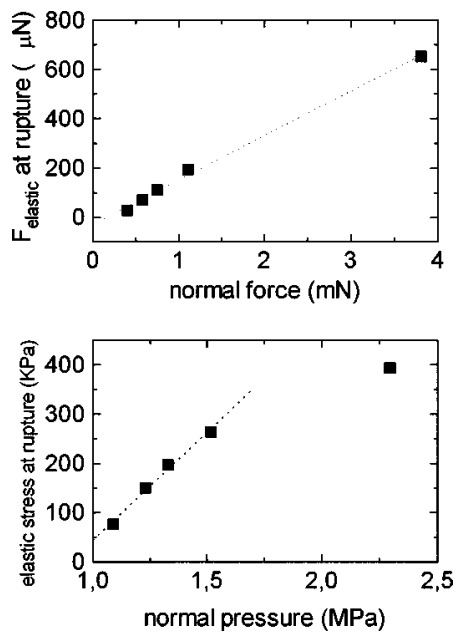

FIG. 7. Elastic force at the rupture point plotted as a function of normal force for the data in Figs. 3 and 4 (top panel). Same data as in top panel except that forces are normalized by contact area to give stress plotted against compressive pressure (bottom panel).

with the normal force as shown in Fig. 7 (top panel). However, calculation of a static friction coefficient from this graph would be misleading due to increase in contact area with increasing normal force.

The peak elastic stress (elastic force normalized by contact area) and the normal pressure (normal force normalized by the contact area) were calculated using estimated contact areas as described in Sec. II. To eliminate the effect of contact area in friction coefficient determination, we plot in Fig. 7 (bottom panel) the elastic stress at rupture against the normal pressure. Except for the data point at the maximum applied pressure, elastic stress at rupture increased linearly with normal pressure. The dotted line is a linear fit to the data and gives a static friction coefficient of 0.44 .

\section{E. Viscous stress in sliding friction regime (kinetic friction)}

In the nonlinear sliding friction regime, viscous force dominated over the elastic force as seen in Fig. 6. At constant oscillation frequency, both elastic and viscous forces were nearly independent of deformation amplitude after the transition. This was attributed in earlier studies ${ }^{9,29}$ to sliding along an interface. In the sliding friction regime, the velocity is the critical parameter. Therefore, in discussion of nonlinear response, we will concentrate on the dominating viscous shear force $F_{v}$ as a function of peak velocity $v_{\max }$, the product of oscillation amplitude and frequency. Figure 8 shows $F_{v}$ as a function of $v_{\max }$ for a 3 ML thick film at varying normal forces. The normal force increased by $0.40 \mathrm{mN}$ from the bottom to the top curve where the onset of discontinuous transition, as indicated by the arrow in Fig. 8, was observed. Within experimental accuracy, the transition from linear to nonlinear response was gradual for the lowest three curves in open symbols. For all the curves the response was linear at low velocities. $F_{v}$ was proportional to $v_{\max }$, giving a slope of 1 in the $\log -\log$ plot. The deviation from a slope of 1 increased with increasing normal force as discussed in Sec. III C. After the transition, in the nonlinear response regime, 


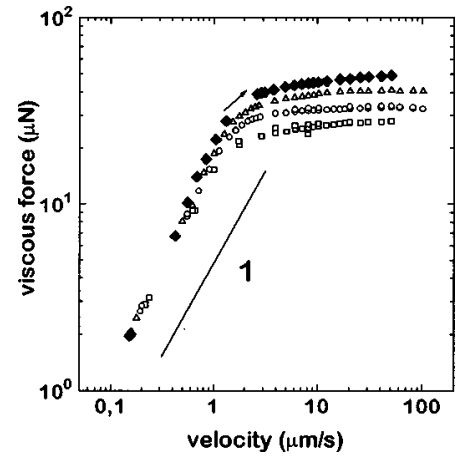

FIG. 8. Viscous force plotted as a function of peak velocity for a film of thickness $3 \mathrm{ML}$ at various normal forces. The normal force increased approximately $0.40 \mathrm{mN}$ from the bottom to the top curve, at which point the onset of a discontinuous transition from stick to slip was observed, as indicated by the arrow.

$F_{v}$ was nearly independent of velocity. It is worth emphasizing that, had the measurements been carried out at variable frequency, the data measured at different frequencies would reduce on a velocity scale only provided that the deformation amplitude greatly exceeded the film thickness. The criteria under which effective velocities (or shear rates) reduce data measured in an oscillatory shear experiment have been discussed elsewhere. ${ }^{8}$

The velocity-independent viscous shear force (stress) is known as the limiting viscous shear force (stress) ${ }^{7}$ and is associated with the kinetic friction. The data showing gradual transitions in Fig. 8 show velocity-independent viscous shear force within experimental accuracy. The discontinuous transition in Fig. 8 showed slight velocity dependence of viscous shear force in the nonlinear regime, with slopes less than 0.1 on $\log -\log$ plots. The limiting viscous shear force values that will be discussed below were determined by averaging the data points in the nearly flat portion of the curves. The significant deviations at the largest velocities, as shown in Fig. 6, will be discussed below.

The logarithm of the limiting viscous shear force is plotted in Fig. 9 (top panel) as a function of the number of molecular layers between the confining surfaces. The scatter of the data at 2 and 3 ML is due to values obtained at different normal pressures. Limiting viscous force increased exponentially with decreasing number of molecular layers. The dotted line is a linear fit to the data and has a slope of 0.44 corresponding to an exponential decay length of $1 \mathrm{ML}$ $(0.44 \ln 10)$. This correlates quite well with the exponential decay length of the structural force oscillations normal to the confining surfaces. ${ }^{34}$ This is understandable because the data were taken in the direction of decreasing film thickness, and so the system was close to the peak of force oscillations at each film thickness. The same exponential decay length for the amplitude of oscillatory normal force and the limiting shear force indicates a linear relationship between the normal force and the limiting viscous shear force. Indeed, Fig. 9 (bottom panel) shows that this linear relationship holds quite well for the data of Fig. 3, giving a slope of 0.14.

The limiting shear stress (the ratio of the shear force to contact area) and the normal pressure (the ratio of the normal force to contact area) were calculated using estimated contact
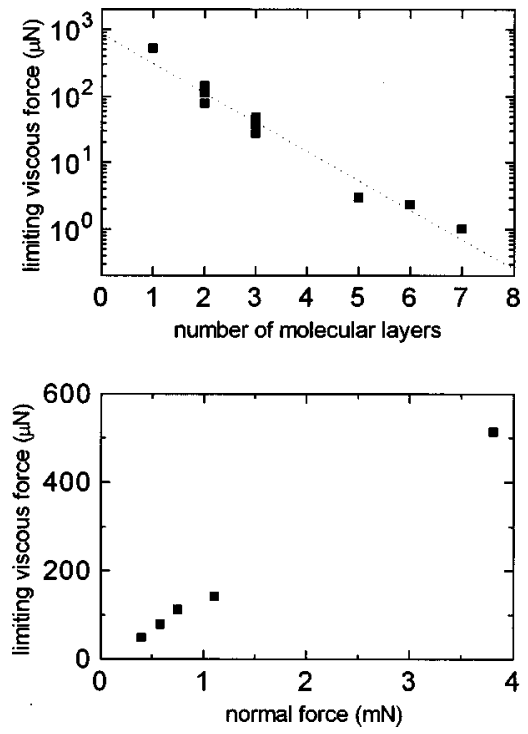

FIG. 9. Semilogarithmic plot of the limiting viscous force against the film thickness, showing that the limiting viscous form increased exponentially with decreasing film thickness. The dotted line is a linear fit to the data giving an exponential decay length of 1 molecular diameter (top panel). The limiting viscous force for the data of Fig. 3 showing linearity with the normal force (bottom panel).

areas. The limiting shear stress was plotted in Fig. 10 against the normal pressure for the data of Fig. 8 (bottom panel). The dashed line is a linear fit to the data also giving a slope of 0.14 that is equal to the kinetic friction coefficient.

\section{F. Upturn in the viscous shear force}

The thinnest films under the largest normal forces indicated an increase of the viscous shear force at the largest velocities, as seen Fig. 6. Immediately after the discontinuous transition where the response switched from being predominantly elastic to predominantly viscous, both the viscous and the elastic forces were nearly independent of the velocity. After a small peak, the elastic force decreased sharply with increasing velocity in the region where the viscous force started increasing. This upturn in the viscous shear force was not due to degradation of the surfaces or the molecules. The system returned reversibly back to the elastic structure with decreasing velocity. Similar behavior was previously observed with confined branched molecules of squalane. ${ }^{9,24,29}$ Under extreme conditions of confinement and

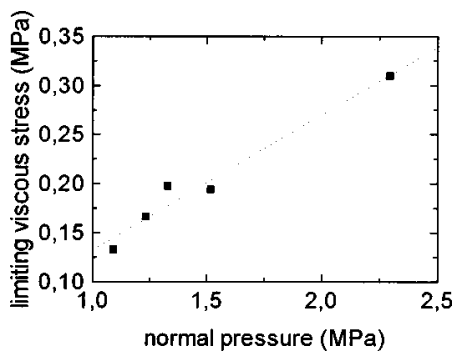

FIG. 10. The force data of Fig. 8 normalized by the contact area, the latter estimated assuming a Hertzian contact. With this normalization, limiting viscous stress is plotted against normal pressure. The dotted line is a linear fit giving a friction coefficient of 0.14 . 
significant elasticity, it was even possible for the surfaces to jump from linear-response regime to the increasing force region in the nonlinear regime. ${ }^{24}$ Such behavior was accompanied by strong hysteresis and significant stress fluctuations in the reverse direction.

\section{DISCUSSION AND CONCLUSIONS}

In the present experimental study of lubricated friction in a model system, we have systematically investigated the effect of lubricating film thickness on both the linear and the nonlinear oscillatory shear response of the confined molecules, and related these to static and kinetic friction coefficients of the system. Since this work was completed ${ }^{25}$ we have learned of another recent study that addressed some aspects of this problem (especially the pressure dependence of friction). ${ }^{35}$ It is difficult to make a quantitative comparison because very large sliding velocities were invariably involved in that study, so that shear stresses had to be inferred from patterns of stick-slip motion during the course of sliding, though these may also be influenced by spring stiffness of the experimental device. ${ }^{35}$ Here we have focused instead on very small deformation amplitudes in order to examine explicitly the transition between the static and kinetic states.

The nature of the transition from static to kinetic friction is surely directly related to the structure of the confined film at rest, and this structure at rest can be probed by lowamplitude vibrations. The resulting linear response, as shown in Fig. 1, showed a monotonic increase in the elasticity (storage modulus, $G^{\prime}$ ) of the confined film with decreasing film thickness. The crossover from predominantly viscous to predominantly elastic response with decreasing film thickness depended on the oscillation frequency. The crossover film thickness decreased with decreasing oscillation frequency. The reciprocal of the angular oscillation frequency at crossover is a measure of the longest relaxation time of the molecules at the crossover film thickness. The data of Fig. 1 indicate a relaxation time of $\approx 16 \mathrm{~ms}$ for a 4 ML thick film and a relaxation time of $\approx 0.6 \mathrm{~ms}$ for a $9 \mathrm{ML}$ thick film. The linear response was predominantly elastic below the crossover film thickness, because the film was sheared faster than it could relax. The loss modulus $G^{\prime \prime}$ showed a peak at a film thickness of approximately $3 \mathrm{ML}$ for a large range of oscillation frequencies $(0.25-250 \mathrm{~Hz}) .{ }^{23}$ Below a film thickness of $3 \mathrm{ML}, G^{\prime}$ was an order of magnitude larger than $G^{\prime \prime}$ indicating the formation of significantly elastic equilibrium structure.

Interpretation of the origins and nature of enhanced elasticity in confined simple fluids has long been debated. . $^{3,8,35,36}$ In a separate study, we have recently studied molecularly thin OMCTS films as a function of film thickness and oscillation frequency ${ }^{23}$ and showed that the longest relaxation time in linear response measurements increased by over four orders of magnitude with decreasing film thickness, below the film thickness of $8 \mathrm{ML}$. The superposition of the frequency spectra taken at different film thickness suggested two sets of widely separated relaxation times and the interpretation of a glass-like transition. Based on only nonlinear measurements, ${ }^{35,36}$ the origin of elasticity has also been attributed to a liquid-to-solid phase transition. A definitive resolution of the apparent discrepancy does not seem possible at this point, but it is our view that measurements of linear responses are most appropriate for the purpose of making inferences about the equilibrium structure of a confined film.

In the present experiments, the transition from linear to nonlinear response was evidently associated with significant changes in the structure of the confined molecules. This transition (which occurred with increasing deformation amplitude) was gradual and reversible without hysteresis down to a film thickness of $3 \mathrm{ML}$ (Fig. 2). With increasing normal force at $3 \mathrm{ML}$ (onset of significant elasticity in the linear response data of Fig. 1), the nature of the transition switched to being discontinuous (Figs. 3 and 4); and the transition was still reversible, but hysteretic. Although the linear response of the system crossed over to being predominantly elastic at a film thickness of $9 \mathrm{ML}$ at $250 \mathrm{~Hz}$, no change in the nature of the transition from linear to nonlinear response was observed down to a film thickness of 3 ML. It would be misleading to conclude an abrupt change in the equilibrium structure of the confined film, with decreasing film thickness, based only on the data of Figs. 2-4. As shown in Fig. 8, the transition from linear to nonlinear response switched from being gradual to discontinuous at a film thickness of $3 \mathrm{ML}$ with increasing normal force.

Based on computer simulations $s^{3,14}$ and on the similarity of observed nonlinear response of significantly elastic OMCTS films to that of confined branched molecules and selfassembled monolayers on mica, ${ }^{9,29}$ we conclude that the mechanism of transition from linear to nonlinear response was slippage along an interface. Globular OMCTS molecules form layers parallel to the confining surfaces and the interface between these layers form well-defined slip planes. Strong interaction of the molecules with the mica surface decreases the probability of slip at the solid boundary compared to inner slip planes and the two layers interacting directly with the two mica surfaces can be considered as immobilized. Then, for films equal to $3 \mathrm{ML}$ or thicker, there are two or more interfaces between layered molecules that can accommodate slippage. The distribution of shear stress to two or more slip planes results in small displacements at each slip plane (discretization of uniform velocity profile at the slip planes) and a gradual transition from linear to nonlinear response. A similar gradual transition and localized slip at shear bands during nonlinear shear response were observed in liquid lubricants under pressure. ${ }^{37}$ In that study of thick lubricant films, the number of shear bands increased with shear rate from as few as one at the onset of nonlinear response until the shear region was filled with bands. Note that in our molecularly thin films, number of slip planes were limited by the number of interfaces between molecular layers (only two slip planes at a film thickness of $3 \mathrm{ML}$ ).

The observation of the onset of discontinuous transition with increasing normal force at a film thickness of $3 \mathrm{ML}$ suggests the role of the in-plane structure of the intermediate layer, which lacked time to relax during the period of the deformation. The transition from linear to nonlinear response was then associated with rupture of this elastic structure and resulted in a predominantly viscous response in the nonlinear 
region. As the structure yielded the elastic strength decreased and the film could not support the applied shear force any more. The apparatus itself (the piezoelectric bimorphs used to shear the upper surface) could not support this force either, and the surfaces jumped to larger relative displacements (larger velocities at constant oscillation frequency).

Two qualitatively different forms of force-velocity relation were also obtained in a theoretical study of a model in which an elastic block slides on a flat substrate with a single monolayer of lubricant molecules separating the block from the substrate. ${ }^{38,39}$ The gradual transition without hysteresis was associated with a two-dimensional fluid state of the monolayer while a solid state pinned by the substrate resulted in a critical stress to start sliding, discontinuity in sliding velocity, and hysteresis. A general explanation for hysteresis was given in terms of different critical velocities for the formation and rupture of pinned elastic islands. ${ }^{40}$

The in-plane structure of the confined film also plays a role in the observed characteristic length scales of Fig. 5. The deformation amplitude at the onset of yield being constant at $3 \AA$ independent of film thickness and normal force suggests that it should be specific to OMCTS molecules. Considering that OMCTS molecules have a diameter of $\sim 9 \AA$, the elastic response below a deformation amplitude of $3 \AA$ should correspond to tumbling motion of an OMCTS molecule in the potential well formed by two underlying molecules.

Long ago, Frenkel ${ }^{41}$ suggested a simple way to estimate the force to produce a shear displacement of two planes of atoms over one another. In this now well-known analysis, ${ }^{42}$ the lattice becomes unstable at a shear displacement of $1 / 2 \pi$ $\approx 0.16$. A regular lattice packing is not required to reach shear instability, however; it is now known that disorderly arrangements of granular materials, and molecular glasses as well, display similar shear instability, as anticipated by the pioneering ideas of Reynolds ${ }^{43}$ on shear-induced dilation. When one considers that the characteristic dimension of the OMCTS molecule studied here was $\approx 9 \AA$, it is quite provocative to conclude that the critical strain of $3 / 9 \approx 0.3$ was so close to the expectation from these simple ideas.

The approach to the constant value of deformation amplitude at yield with increasing normal force indicate increasing rigidity of the confined system, and yield by a process that more nearly approaches the yield of a relatively well-ordered single layer of confined molecules.

The increase in the contact area with increasing normal force has been shown to contribute significantly to the measured increase of elastic force at rupture. After normalizing the friction force and the normal force by the contact area, the static friction coefficient was found to be 0.44 and kinetic friction coefficient to be 0.14 . In other words, the elastic force needed to rupture the system increased more rapidly with applied pressure than did the viscous force needed to shear it at high speed. This difference between the static and kinetic friction coefficients is what makes the discontinuous transition from static to kinetic friction critical in boundary lubrication. Because of the sudden decrease in the friction force at the start-up of motion, the surfaces jump to larger displacements with respect to each other. This might degrade the system, especially the confining solid surfaces, in time.
After the transition from static to kinetic friction, viscous shear force dominated over the elastic force and both were nearly independent of the velocity. This velocityindependent stress region may correspond to sliding of two surface-immobilized layers on top of each other. The elastic component remained significant until very large velocities. The decrease of the elastic stress and the increase of the viscous stress at the largest velocities would then be due to rupture of the elastic structure in the immobilized layers. Such upturn in the viscous stress was also typical for confined branched molecules. ${ }^{29,30}$ Not only the saturation of the viscous shear stress at a constant value, but also the onset of increase in the shear stress at the largest shear rates have been observed in many different systems such as polymers, ${ }^{5}$ colloids, ${ }^{21}$ lubricants under pressure, $^{7}$ and worm-like micelles. ${ }^{18,19}$ Though different mechanisms are responsible in the molecular scale for different systems, slippage is common to them.

For simple liquids with regularly shaped molecules, the structural force profile as a function of surface separation can be described as an exponentially decaying cosine function with a decay length and periodicity equal to the mean molecular diameter. ${ }^{34,44}$ The limiting shear force also showed an exponential dependence on the number of molecular layers, as shown in Fig. 9 (top panel), with a decay length of one molecular diameter. This implies a linear relationship between the limiting shear force and the normal force, provided that the system is close to the peak of force oscillations. This was the case in our measurements, because the measurements were done in the direction of decreasing surface separation (increasing normal force). Figure 9 (bottom panel) shows the linearity between the limiting viscous shear force and the normal force. The linearity also holds when the forces are normalized by the contact area, as seen in Fig. 10, giving a well-defined kinetic friction coefficient.

In summary, the close relationship between the microscopic structure in the lubricating film and the macroscopic frictional behavior of a model lubricated system was investigated in this study. The formation of elastic structure between the confining surfaces not only changed the transition from gradual to discontinuous, but also affected the deformation amplitude necessary for the transition. The friction showed well-defined dependence on the film thickness, the normal force and the normal pressure.

\section{ACKNOWLEDGMENTS}

This work was supported by the National Science Foundation (Tribology Program) with generous contributions from Exxon Research and Engineering Corp. and Ford Corp.

\footnotetext{
${ }^{1}$ Physics of Sliding Friction, edited by B. N. J. Persson and E. Tosatti (Kluwer Academic, Dordrecht, 1996).

${ }^{2}$ Dynamics in Small Confining Systems, edited by J. M. Drake, J. Klafter, S. M. Troian, and R. Kopelman (Materials Research Society, Pittsburgh, 1995).

${ }^{3}$ B. Bhushan, J. N. Israelachvili, and U. Landman, Nature (London) 374, 607 (1995).

${ }^{4}$ D. Dowson, History of Tribology (Longman, London, 1979).

${ }^{5}$ J. D. Ferry, Viscoelastic Properties of Polymers, 3rd ed. (Wiley, New York, 1980)
} 
${ }^{6}$ H.-W. Hu, G. A. Carson, and S. Granick, Phys. Rev. Lett. 66, 2758 (1991).

${ }^{7}$ S. Bair and W. O. Winer, J. Lubr. Technol. 101, 251 (1979).

${ }^{8}$ S. Granick, Science 253, 1374 (1991)

${ }^{9}$ G. Reiter, A. L. Demirel, and S. Granick, Science 263, 1741 (1994).

${ }^{10}$ R. G. Larson, K. I. Winey, S. S. Patel, H. Watanabe, and R. Bruinsma, Rheol. Acta 32, 245 (1993).

${ }^{11}$ S. Hess, Physica A 118, 79 (1983).

${ }^{12}$ S. G. Hatzikiriakos and J. M. Dealy, J. Rheol. 36, 45 (1992).

${ }^{13}$ D. S. Kalika and M. Denn, J. Rheol. 31, 815 (1987).

${ }^{14}$ P. A. Thompson, G. S. Grest, and M. O. Robbins, Phys. Rev. Lett. 68, 3448 (1992).

${ }^{15}$ H. Watanabe, T. Kotaka, T. Hashimoto, M. Shibayama, and H. Kawai, J. Rheol. 26, 153 (1982)

${ }^{16}$ T. Ohta, Y. Enomoto, J. L. Harden, and M. Doi, Macromolecules 26, 4928 (1993).

${ }^{17}$ M. Doi, J. Harden and T. Ohta, Macromolecules 26, 4935 (1993).

${ }^{18}$ N. A. Spenley, M. E. Cates, and T. C. B. McLeish, Phys. Rev. Lett. 71, 939 (1993)

${ }^{19}$ F. Lequeux, Curr. Opin. Colloid Interface Sci. 1, 341 (1996).

${ }^{20}$ L. A. Archer, D. Ternet, and R. G. Larson, Rheol. Acta 36, 579 (1997).

${ }^{21}$ W. B. Russell, D. A. Savlle, and W. R. Schowalter, Colloidal Dispersions (Cambridge University Press, Cambridge, 1989).

${ }^{22}$ A. M. Kraynik, Annu. Rev. Fluid Mech. 20, 325 (1988).

${ }^{23}$ A. L. Demirel and S. Granick, Phys. Rev. Lett. 77, 2261 (1996).

${ }^{24}$ A. L. Demirel and S. Granick, Phys. Rev. Lett. 77, 4330 (1996).

${ }^{25}$ A. L. Demirel, Ph.D. thesis, University of Illinois, 1996.
${ }^{26}$ J. N. Israelachvili, P. M. McGuiggan, and A. M. Homola, Science 240, 189 (1988).

${ }^{27}$ H. Yoshizawa and J. Israelachvili, J. Phys. Chem. 97, 11300 (1993).

${ }^{28} \mathrm{~J}$. N. Israelachvili, in Handbook of Micro/Nanotribology, edited by B. Bhushan (Chemical Rubber, Boca Raton, 1995), p. 269.

${ }^{29}$ G. Reiter, A. L. Demirel, J. Peanasky, L. Cai, and S. Granick, J. Chem. Phys. 101, 2606 (1994).

${ }^{30}$ S. Granick, A. L. Demirel, L. Cai, and J. Peanasky, Isr. J. Chem. 35, 75 (1995).

${ }^{31}$ J. Peachey, J. Van Alsten, and S. Granick, Rev. Sci. Instrum. 62, 463 (1991).

${ }^{32}$ S. Onogi, T. Masuda, and T. Matsumoto, Trans. Soc. Rheol. 14, 275 (1970).

${ }^{33}$ A. J. Giacomin and J. G. Oakley, Rheol. Acta 32, 328 (1993).

${ }^{34}$ R. Horn and J. N. Israelachvili, J. Chem. Phys. 75, 1400 (1981).

${ }^{35}$ E. Kumacheva and J. Klein, J. Chem. Phys. 108, 7010 (1998).

${ }^{36}$ E. Kumacheva and J. Klein, Science 269, 5225 (1995).

${ }^{37}$ S. Bair, F. Qureshi, and W. O. Winer, J. Tribol. 115, 507 (1993).

${ }^{38}$ B. N. J. Persson, Phys. Rev. Lett. 71, 1212 (1993).

${ }^{39}$ B. N. J. Persson, Phys. Rev. B 48, 18140 (1993).

${ }^{40}$ B. N. J. Persson, Phys. Rev. B 50, 4771 (1994).

${ }^{41}$ J. Frenkel, Z. Phys. 37, 572 (1926).

${ }^{42}$ C. Kittel, Introduction to Solid State Physics, 3rd ed. (Wiley, New York, 1968).

${ }^{43}$ O. Reynolds, Philos. Mag. 20, 469 (1885).

${ }^{44}$ S. Tarazona and L. Vincente, Mol. Mater. 56, 557 (1985). 